J. Nonlinear Var. Anal. 3 (2019), No. 2, pp. 181-187

Available online at http://jnva.biemdas.com

https://doi.org/10.23952/jnva.3.2019.2.07

\title{
UNIFIED FORMS OF FIXED POINT THEOREMS AND MINIMAX INEQUALITIES
}

\author{
NAOKI SHIOJI \\ Department of Mathematics, Faculty of Engineering, Yokohama National University, \\ Tokiwadai, Hodogaya, Yokohama 240-8501, Japan
}

\begin{abstract}
We show a unified form of two fixed point theorems and a unified form of a fixed point theorem and a minimax inequality. We also apply our results to show the existence of solutions of generalized quasi-variational inequalities.

Keywords. Fixed point theorems, Minimax inequalities, Variational inequalities.
\end{abstract}

2010 Mathematics Subject Classification. 47H10, 47H05.

\section{INTRODUCTION}

Let $E$ be a locally convex Hausdorff topological vector space, and let $X$ be a nonempty subset of $E$. We denote by $E^{\prime}$ the space of all continuous linear functionals on $E$. Let $S: X \rightarrow 2^{X}$ and $T: X \rightarrow 2^{E^{\prime}}$ be set-valued mappings, i.e., for each $x \in X, S x$ is a subset of $X$ and $T x$ is a subset of $E^{\prime}$. A generalized quasi-variational inequality problem $([3,10])$ is to find $x \in X$ such that

$x \in S x$, and there exists $u \in T x$ such that $\operatorname{Re}\langle u, y-x\rangle \geq 0$ for each $y \in S x$.

Shih and Tan [10] proved theorems concerning this problem. Two of them are Corollaries 3.1 and 3.2 in this paper. From their results, we can deduce the Fan-Glicksberg fixed point theorem and the existence of a solution of a variational inequality. That is, their results are combinations of a fixed point theorem and the existence of a solution of a variational inequality.

Motivated by their results, we will show some unified forms of fixed point theorems and minimax inequality theorems. Our results are related to the following celebrated theorems in the fixed point theory. The following is the Fan-Glicksberg fixed point theorem $[4,8]$.

Theorem 1.1 (Fan and Glicksberg). Let X be a compact, convex subset of a locally convex Hausdorff topological vector space. Let $S: X \rightarrow 2^{X}$ be an upper semicontinuous set-valued mapping such that, for each $x \in X$, Sx is nonempty, closed and convex. Then there is $x \in X$ such that $x \in S x$.

The following fixed point theorem was obtained by Ben-El-Mechaiekh, Deguire and Granas [1], which is a generalization of Fan-Browder's fixed point theorem [2, 5].

Theorem 1.2 (Ben-El-Mechaiekh, Deguire and Granas). Let $X$ be a nonempty, compact, convex subset of a Hausdorff topological vector space. Let $A, B: X \rightarrow 2^{X}$ be set-valued mappings such that

(1) for each $x \in X, B x \subset A x$ and $B x$ is a relatively open subset of $X$,

E-mail address: shioji-naoki-jh@ynu.ac.jp.

Received December 31, 2018; Accepted April 10, 2019.

(C)2019 Journal of Nonlinear and Variational Analysis 
(2) for each $y \in X, B^{-1} y$ is nonempty and $A^{-1} y$ is convex.

Then there exists $x \in X$ such that $x \in A x$.

The following minimax inequality theorem was obtained by Yen [12], which is a generalization of Fan's one [6]. Shih and Tan applied it to obtain their results including Corollaries 3.1 and 3.2 below.

Theorem 1.3 (Yen). Let $X$ be a compact, convex set in a Hausdorff topological vector space. Let $f, g: X \rightarrow \mathbb{R}$ such that

(1) for each $x \in X, g(x, \cdot)$ is lower semicontinuous on $X$,

(2) for each $y \in X, f(\cdot, y)$ is quasi-concave on $X$,

(3) $g(x, y) \leq f(x, y)$ for each $(x, y) \in X \times X$.

Then the minimax inequality

$$
\min _{y \in X} \sup _{x \in X} g(x, y) \leq \sup _{x \in X} f(x, x)
$$

holds.

In this article, we show a unified form of two fixed point theorems and a unified form of a fixed point theorem and a minimax inequality, and we apply our results to show the existence of solutions of generalized quasi-variational inequalities.

\section{PRELIMINARIES}

We recall some definitions. Let $X$ and $Y$ be Hausdorff topological spaces, and let $S: X \rightarrow 2^{Y}$ be a set-valued mapping such that, for each $x \in X, S x$ is a nonempty subset of $Y$. We say $S$ is upper semicontinuous if, for each point $x_{0} \in X$ and each open set $V$ in $Y$ with $S x_{0} \subset V$, there is a neighborhood $U$ of $x_{0}$ in $X$ such that $S x \subset V$ for each $x \in U$. If $Y$ is compact and $S x$ is closed for each $x \in X$, then $S$ is upper semicontinuous if and only if the graph $\{(x, y) \in X \times Y: y \in S x\}$ of $S$ is closed in $X \times Y$. We say $S$ is lower semicontinuous if, for each point $x_{0} \in X$ and each open set $V$ in $Y$ with $S x_{0} \cap V \neq \emptyset$, there is a neighborhood $U$ of $x_{0}$ in $X$ such that $S x \cap V \neq \emptyset$ for each $x \in U$. If $S$ is lower semicontinuous, a net $\left\{x_{\alpha}\right\} \subset X$ converges to $x_{0} \in X$ and $y_{0} \in S x_{0}$, then there exist a subnet $\left\{x_{\alpha_{\beta}}\right\}$ of $\left\{x_{\alpha}\right\}$ and a net $\left\{y_{\beta}\right\}$ such that $y_{\beta} \in S x_{\alpha_{\beta}}$ for each $\beta$ and $\left\{y_{\beta}\right\}$ converges to $y_{0}$. We say $S$ is continuous if it is upper semicontinuous and lower semicontinuous.

For a set-valued mapping $A: X \rightarrow 2^{Y}$, we denote by $A^{-1} y$ the set $\{x \in X: y \in A x\}$ for each $y \in Y$. In the case that $X \subset Y$ and $Y$ is convex, we say a set-valued mapping $G: X \rightarrow 2^{Y}$ is a KKM-map if

$$
\operatorname{co}\left\{x_{1}, \ldots, x_{n}\right\} \subset \bigcup_{i=1}^{n} G x_{i} \text { for each finite subset }\left\{x_{1}, \ldots, x_{n}\right\} \text { of } X,
$$

where $\operatorname{co}\left\{x_{1}, \ldots, x_{n}\right\}$ denotes the convex hull of $\left\{x_{1}, \ldots, x_{n}\right\}$.

We recall Fan's generalization [7] of the Knaster-Kuratowski-Mazurkiewicz theorem [9].

Theorem 2.1 (Fan). Let $X, Y$ be subsets of a Hausdorff topological vector space such that $\emptyset \neq X \subset Y$ and $Y$ is convex. Let $G: X \rightarrow 2^{Y}$ be a KKM-map such that, for each $x \in X$, Gx is a relatively closed subset of $Y$. If there is a nonempty subset $X_{0}$ of $X$ such that $\bigcap_{x \in X_{0}} G x$ is compact and $X_{0}$ is contained in a compact, convex subset of $Y$, then $\bigcap_{x \in X} G x \neq \emptyset$. 


\section{MAin RESUlTS AND APPLICATIONS}

We state our unified form of the Fan-Glicksberg fixed point theorem and Ben-El-Mechaiekh-DeguireGranas's fixed point theorem. We note that we can derive the Tychonoff fixed point theorem [11] by considering $S: X \rightarrow X$ is a single-valued continuous mapping and $A x=B x=X$ for each $x \in X$. We also note that it has a similar form to Theorem 1.2.

Theorem 3.1. Let $X$ be a nonempty, compact, convex subset of a locally convex Hausdorff topological vector space. Let $S: X \rightarrow 2^{X}$ be a continuous set-valued mapping such that, for each $x \in X, S x$ is a nonempty, closed, convex subset of $X$. Let $A, B: X \rightarrow 2^{X}$ be set-valued mappings such that

(1) for each $x \in X, x \in B x, B x \subset A x$ and $X \backslash B x$ is convex,

(2) for each $y \in X, A^{-1} y$ is closed and convex,

(3) A is upper semicontinuous.

Then there exists $x \in X$ such that $x \in S x$ and $S x \subset A x$.

Proof. We define a set-valued mapping $T: X \rightarrow 2^{X}$ by

$$
T x=\{z \in S x: S x \subset A z\} \quad \text { for each } x \in X .
$$

Let $x \in X$. Since $T x=S x \cap \bigcap_{y \in S x} A^{-1} y, T x$ is closed and convex. We will show that $T x$ is nonempty. We claim that the set-valued mapping $z \mapsto S x \cap B^{-1} z: S x \rightarrow 2^{S x}$ is a KKM-map. Assume that the claim does not hold. Then there exist $\left\{z_{1}, \ldots, z_{n}\right\} \subset S x$ and $z \in \operatorname{co}\left\{z_{1}, \ldots, z_{n}\right\}$ such that $z \notin \bigcup_{i=1}^{n}\left(S x \cap B^{-1} z_{i}\right)$. We may assume that $z=\sum_{i=1}^{n} \lambda_{i} z_{i}$ with $\lambda_{i}>0$ for each $i=1, \ldots, n$ and $\sum_{i=1}^{n} \lambda_{i}=1$. Since $S x$ is convex, we have $z \in S x$. Hence $z_{i} \in X \backslash B z$ for each $i=1, \ldots, n$. Since $X \backslash B z$ is convex, we obtain $z \notin B z$, which is a contradiction. Thus we have shown the claim. Since $S x \cap B^{-1} z \subset S x \cap A^{-1} z$ for each $z \in S x$, the set-valued mapping $z \mapsto S x \cap A^{-1} z: S x \rightarrow 2^{S x}$ is also a KKM-map. Hence, by Theorem 2.1, we have $T x=S x \cap \bigcap_{y \in S x} A^{-1} y \neq \emptyset$. Thus we have shown that $T x$ is nonempty.

Next, we show that $T$ is upper semicontinuous. Let $\left\{\left(x_{\alpha}, z_{\alpha}\right)\right\} \subset X \times X$ be a net such that $z_{\alpha} \in T x_{\alpha}$ for each $\alpha$ and $\left\{\left(x_{\alpha}, z_{\alpha}\right)\right\}$ converges to $(x, z) \in X \times X$. Since $S$ is upper semicontinuous, we have $z \in S x$. Let $y$ be an arbitrary element of $S x$. Since $S$ is lower semicontinuous, there exist a subnet $\left\{x_{\alpha_{\beta}}\right\}$ of $\left\{x_{\alpha}\right\}$ and a net $\left\{y_{\beta}\right\}$ such that $y_{\beta} \in S x_{\alpha_{\beta}}$ for each $\beta$ and $\left\{y_{\beta}\right\}$ converges to $y$. By the upper semicontinuity of $S$ and $A$, we have $y \in A z$. Since $y$ is an arbitrary element of $S x$, we have $S x \subset A z$, which yields $z \in T x$. Thus we have shown that $T$ is upper semicontinuous. Hence by Theorem 1.1, we find that $T$ has a fixed point $x \in X$, i.e., $x \in S x$ and $S x \subset A x$.

Let $X$ be a nonempty subset of a locally convex Hausdorff topological vector space $E$, and let $T$ be a set-valued mapping of $X$ into $E^{\prime}$ such that, for each $x \in X, T x$ in nonempty. We say $T$ is monotone if

$$
\operatorname{Re}\langle u-v, x-y\rangle \geq 0 \quad \text { for each } x, y \in X \text { and for each } u \in T x, v \in T y .
$$

Corollary 3.1 (Shih and Tan). Let $E$ be a locally convex Hausdorff topological vector space, and let $X$ be a nonempty, compact, convex subset of $E$. Let $S: X \rightarrow 2^{X}$ be a continuous set-valued mapping such that, for each $x \in X$, Sx is a nonempty, closed, convex subset of $X$. Let $T: X \rightarrow 2^{E^{\prime}}$ be a monotone, lower semicontinuous set-valued mapping from $X$ to the strong topology of $E^{\prime}$ such that, for each $x \in X, T x$ is a nonempty subset of $E^{\prime}$. Then there exists $x \in X$ such that

$$
x \in S x \quad \text { and } \quad \inf _{u \in T x} \operatorname{Re}\langle u, y-x\rangle \geq 0 \quad \text { for each } y \in S x .
$$


Proof. We define set-valued mappings $A, B: X \rightarrow 2^{X}$ by

$$
\begin{array}{ll}
A x=\left\{y \in X: \inf _{v \in T y} \operatorname{Re}\langle v, y-x\rangle \geq 0\right\} & \text { for each } x \in X, \\
B x=\left\{y \in X: \sup _{u \in T x} \operatorname{Re}\langle u, y-x\rangle \geq 0\right\} & \text { for each } x \in X,
\end{array}
$$

respectively. It is easy to see that, for each $x \in X, x \in B x \subset A x$ and $X \backslash B x$ is convex, and ,for each $y \in X, A^{-1} y$ is closed and convex. Since $T$ is lower semicontinuous from the topology of $E$ to the strong topology of $E^{\prime}, A$ is upper semicontinuous. By Theorem 3.1, there exists $x \in X$ such that

$$
x \in S x \quad \text { and } \quad \inf _{v \in T y} \operatorname{Re}\langle v, y-x\rangle \geq 0 \quad \text { for each } y \in S x .
$$

We will show

$$
\inf _{u \in T x} \operatorname{Re}\langle u, z-x\rangle \geq 0 \quad \text { for each } z \in S x .
$$

Let $z \in S x$. Since $x \in S x$ and $S x$ is convex, we have $z_{t}=t z+(1-t) x \in S x$ for each $t \in(0,1)$. So

$$
\inf _{v \in T z_{t}} \operatorname{Re}\langle v, z-x\rangle \geq 0 \quad \text { for each } t \in(0,1) .
$$

By

$$
s \geq t \Leftrightarrow s-t \in(-\infty, 0] \quad \text { for each } s, t \in(0,1),
$$

we consider that $(0,1)$ is a directed set and $\left\{z_{t}\right\}$ is a net. We note that $\left\{z_{t}\right\}$ converges to $x$. Let $u \in T x$. Since $T$ is lower semicontinuous, there exist a subnet $\left\{z_{t_{\alpha}}\right\}$ of $\left\{z_{t}\right\}$ and a net $\left\{u_{\alpha}\right\}$ such that $u_{\alpha} \in T z_{t_{\alpha}}$ for each $\alpha$ and $\left\{u_{\alpha}\right\}$ converges to $u$ in the $\sigma-\left(E^{\prime}, E\right)$ topology of $E^{\prime}$. From (3.2), we have $\operatorname{Re}\left\langle u_{\alpha}, z-x\right\rangle \geq 0$ for each $\alpha$, which yields $\operatorname{Re}\langle u, z-x\rangle \geq 0$. Since $u$ is an arbitrary element of $T x$, we obtain (3.1). This completes the proof.

Let $X$ be a nonempty subset of a locally convex topological vector space $E$. For a set-valued mapping $S: X \rightarrow 2^{E}$ such that, for each $x \in X, S x$ is nonempty, we say $S$ is upper demicontinuous if for each $x_{0} \in X$, for each $\varphi \in E^{\prime}$ and for each $t \in \mathbb{R}$ with $S x \subset\{y \in X: \operatorname{Re} \varphi(y)<t\}$, there exists an neighborhood $U$ of $x_{0}$ in $X$ such that for each $z \in U, S z \subset\{y \in X: \operatorname{Re} \varphi(y)<t\}$.

The following is our unified form of the Fan-Glicksberg fixed point theorem and a minimax inequality.

Theorem 3.2. Let $E$ be a locally convex Hausdorff topological vector space, and let $X$ be a nonempty, paracompact, convex subset of $E$. Let $S: X \rightarrow 2^{X}$ be an upper demicontinuous set-valued mapping such that, for each $x \in X$, Sx is a nonempty, closed, convex subset of $X$. Let $f, g$ be functions of $X \times X$ into $\mathbb{R}$ such that

(1) for each $x \in X, f(x, x) \geq 0$ and $f(x, \cdot)$ is a convex function of $X$ into $\mathbb{R}$,

(2) for each $y \in X, g(\cdot, y)$ is an upper semicontinuous function of $X$ into $\mathbb{R}$,

(3) for each $(x, y) \in X \times X, f(x, y) \leq g(x, y)$.

Suppose further that the set $V_{0}=\left\{x \in X: \inf _{y \in S x} g(x, y)<0\right\}$ is relatively open in $X$ and there exist a nonempty, compact, convex subset $K$ of $X$ and a compact subset $L$ of $X$ such that

(4) for each $x \in X \backslash L, S x \cap K \neq \emptyset$,

(5) for each $x \in(X \backslash L) \cap V_{0}$, there exists $y \in S x \cap K$ with $g(x, y)<0$.

Then there exists $x \in X$ such that

$$
x \in S x \quad \text { and } \quad g(x, y) \geq 0 \quad \text { for each } y \in S x .
$$


Proof. Suppose that the assertion dose not hold. Then, for each $x \in X$, either $x \notin S x$ or there exists $y \in S x$ such that $g(x, y)<0$. In the former case, there exist $p \in E^{\prime} \backslash\{0\}$ and $t \in \mathbb{R}$ such that $\operatorname{Re}\langle p, x\rangle>t>$ $\operatorname{Re}\langle p, y\rangle$ for each $y \in S x$, and in the latter case, it holds $x \in V_{0}$. For each $p \in E^{\prime} \backslash\{0\}$, let

$$
V_{p}=\{x \in X: \text { there exists } t \in \mathbb{R} \text { such that } \operatorname{Re}\langle p, x\rangle>t>\operatorname{Re}\langle p, y\rangle \text { for each } y \in S x\} .
$$

Then $\left\{V_{0}\right\} \cup\left\{V_{p}: p \in E^{\prime} \backslash\{0\}\right\}$ is an open covering of $X$. Since $X$ is paracompact, there exists a continuous partition of unity $\left\{\beta_{0}\right\} \cup\left\{\beta_{p}: p \in E^{\prime} \backslash\{0\}\right\}$ subordinate to this open covering. We define set-valued mappings $F, G: X \rightarrow 2^{X}$ by

$$
\begin{array}{ll}
F y=\left\{x \in X: \beta_{0}(x) f(x, y)+\sum_{p \in E^{\prime} \backslash\{0\}} \beta_{p}(x) \operatorname{Re}\langle p, y-x\rangle \geq 0\right\} & \text { for each } y \in X, \\
G y=\left\{x \in X: \beta_{0}(x) g(x, y)+\sum_{p \in E^{\prime} \backslash\{0\}} \beta_{p}(x) \operatorname{Re}\langle p, y-x\rangle \geq 0\right\} & \text { for each } y \in X .
\end{array}
$$

We show that $F$ is a KKM-map. If not, then there exists a finite subset $\left\{y_{1}, \cdots, y_{n}\right\}$ of $X$ and $\left(\lambda_{1}, \ldots, \lambda_{m}\right) \in$ $\mathbb{R}^{m}$ such that $\lambda_{j}>0$ for each $j=1, \ldots, m, \sum_{j=1}^{m} \lambda_{j}=1$ and $\sum_{j=1}^{m} \lambda_{j} y_{j} \notin \bigcup_{j=1}^{m} F y_{j}$. Letting $y=\sum_{j=1}^{m} \lambda_{j} y_{j}$, we have

$$
\beta_{0}(y) f\left(y, y_{j}\right)+\sum_{p \in E^{\prime} \backslash\{0\}} \beta_{p}(y) \operatorname{Re}\left\langle p, y_{j}-y\right\rangle<0 \quad \text { for each } j=1, \cdots, m .
$$

Then

$$
0>\sum_{j=1}^{m} \lambda_{j}\left(\beta_{0}(y) f\left(y, y_{j}\right)+\sum_{p \in E^{\prime} \backslash\{0\}} \beta_{p}(y) \operatorname{Re}\left\langle p, y_{j}-y\right\rangle\right) \geq \beta_{0}(y) f(y, y) \geq 0,
$$

which is a contradiction. Hence $F$ is a KKM-map. Since $F y \subset G y$ for each $y \in X, G$ is also a KKM-map, and for each $y \in X, G y$ is closed. By Theorem 2.1, we can see that if $\bigcap_{y \in K} G y \subset L$, then $\bigcap_{y \in X} G y \neq \emptyset$. So, $\bigcap_{y \in K} G y \backslash L \neq \emptyset$ or $\bigcap_{y \in X} G y \neq \emptyset$. We will obtain a contradiction in each of these cases. First, we consider the case $\bigcap_{y \in X} G y \neq \emptyset$. Let $\hat{x} \in \bigcap_{y \in X} G y$. Then

$$
\beta_{0}(\hat{x}) g(\hat{x}, y)+\sum_{p \in E^{\prime} \backslash\{0\}} \beta_{p}(\hat{x}) \operatorname{Re}\langle p, y-\hat{x}\rangle \geq 0 \quad \text { for each } y \in X .
$$

In the case $\beta_{0}(\hat{x})>0$, we choose $\hat{y} \in S \hat{x}$ such that $g(\hat{x}, \hat{y})<0$. In the case $\beta_{0}(\hat{x})=0$, let $\hat{y}$ be any element of $S \hat{x}$. We note that if $\beta_{p}(\hat{x})>0$ with $p \neq 0$, then $\hat{x} \in V_{p}$, which implies $\operatorname{Re}\langle p, \hat{y}-\hat{x}\rangle<0$. Hence

$$
\beta_{0}(\hat{x}) g(\hat{x}, \hat{y})+\sum_{p \in E^{\prime} \backslash\{0\}} \beta_{p}(\hat{x}) \operatorname{Re}\langle p, \hat{y}-\hat{x}\rangle<0,
$$

which contradicts (3.3).

Next, we consider the case $\bigcap_{y \in K} G y \backslash L \neq \emptyset$. Let $\tilde{x} \in \bigcap_{y \in K} G y \backslash L$. Then $\tilde{x} \in X \backslash L$ and

$$
\beta_{0}(\tilde{x}) g(\tilde{x}, y)+\sum_{p \in E^{\prime} \backslash\{0\}} \operatorname{Re}\langle p, y-\tilde{x}\rangle \geq 0 \quad \text { for each } y \in K .
$$

In the case $\beta_{0}(\tilde{x})>0$, we have $\tilde{x} \in(X \backslash L) \cap V_{0}$ and we choose $\tilde{y} \in S \tilde{x} \cap K$ to satisfy $g(\tilde{x}, \tilde{y})<0$. In the case $\beta_{0}(\tilde{x})=0$, we choose any $\tilde{y} \in S \tilde{x} \cap K$. Hence, we can see

$$
\beta_{0}(\tilde{x}) g(\tilde{x}, \tilde{y})+\sum_{p \in E^{\prime} \backslash\{0\}} \beta_{p}(\tilde{x}) \operatorname{Re}\langle p, \tilde{y}-\tilde{x}\rangle<0,
$$

which contradicts (3.4). Thus, we obtain a contradiction in each case of $\bigcap_{y \in K} G y \backslash L \neq \emptyset$ and $\bigcap_{y \in X} G y \neq \emptyset$. Therefore we have shown that the assertion holds. 
In the case that $X$ is compact, we have the following. We can derive the Fan-Glicksberg fixed point theorem by setting $f=g=0$.

Theorem 3.3. Let $E$ be a locally convex Hausdorff topological vector space, and let $X$ be a nonempty, compact, convex subset of $E$. Let $S: X \rightarrow 2^{X}$ be an upper semicontinuous set-valued mapping such that for each $x \in X$, Sx is a nonempty, closed, convex subset of $X$. Let $f, g$ be functions of $X \times X$ into $\mathbb{R}$ such that

(1) for each $x \in X, f(x, x) \geq 0$ and $f(x, \cdot)$ is a convex function of $X$ into $\mathbb{R}$,

(2) for each $y \in X, g(\cdot, y)$ is an upper semicontinuous function of $X$ into $\mathbb{R}$,

(3) for each $(x, y) \in X \times X, f(x, y) \leq g(x, y)$.

Suppose further that the set $\left\{x \in X: \inf _{y \in S x} g(x, y)<0\right\}$ is relatively open in $X$. Then there exists $x \in X$ such that

$$
x \in S x \quad \text { and } \quad g(x, y) \geq 0 \quad \text { for each } y \in S x .
$$

Let $X$ be a nonempty subset of a locally convex Hausdorff topological vector space $E$. Let $T: X \rightarrow 2^{E^{\prime}}$ such that for each $x \in X, T X$ in nonempty. We say $T$ is lower hemicontinuous if for each line segment $L$ in $E,\left.T\right|_{X \cap L}: X \cap L \rightarrow 2^{E^{\prime}}$ is lower semicontinuous from the topology of $E$ to the $\sigma-\left(E^{\prime} . E\right)$ topology of $E^{\prime}$.

Theorem 3.4. Let $E$ be a locally convex Hausdorff topological vector space, and let $X$ be a nonempty, paracompact, convex subset of $E$. Let $S: X \rightarrow 2^{X}$ be an upper demicontinuous set-valued mapping such that, for each $x \in X, S x$ is a nonempty, closed, convex subset of $X$. Let $T: X \rightarrow 2^{E^{\prime}}$ be monotone and lower hemicontinuous such that, for each $x \in X, T x$ is a nonempty subset of $E^{\prime}$. Suppose further that the set $V_{0}=\left\{x \in X: \inf _{y \in S x} \inf _{v \in T y} \operatorname{Re}\langle v, y-x\rangle<0\right\}$ is relatively open in $X$ and there exist a nonempty, compact, convex subset $K$ of $X$ and a compact subset $L$ of $X$ such that

(1) for each $x \in X \backslash L, S x \cap K \neq \emptyset$,

(2) for each $x \in(X \backslash L) \cap V_{0}$, there exists $y \in S x \cap K$ such that

$$
\inf _{v \in T y} \operatorname{Re}\langle v, y-x\rangle<0 \text {. }
$$

Then there exists $x \in X$ such that

$$
x \in S x \quad \text { and } \quad \inf _{u \in T x} \operatorname{Re}\langle u, y-x\rangle \geq 0 \quad \text { for each } y \in S x .
$$

Proof. Define functions $f, g$ on $X \times X$ by

$$
f(x, y)=\sup _{u \in T x} \operatorname{Re}\langle u, y-x\rangle \quad \text { and } \quad g(x, y)=\inf _{v \in T y} \operatorname{Re}\langle v, y-x\rangle
$$

for each $(x, y) \in X \times X$. Since $T$ is monotone and nonempty-valued, we have $f \leq g$. Hence $f$ and $g$ are real-valued. By Theorem 3.2, there exists $x \in X$ such that $x \in S x$ and $\inf _{v \in T y} \operatorname{Re}\langle v, y-x\rangle \geq 0$ for each $y \in S x$. By the same lines as those in the proof of Theorem 3.1, we have

$$
\inf _{u \in T x} \operatorname{Re}\langle u, y-x\rangle \geq 0 \quad \text { for each } y \in S x .
$$

As a direct consequence, we have the following. 
Corollary 3.2 (Shih and Tan). Let $E$ be a locally convex Hausdorff topological vector space, and let $X$ be a nonempty, compact, convex subset of $E$. Let $S: X \rightarrow 2^{X}$ be an upper semicontinuous set-valued mapping such that, for each $x \in X$, Sx is a nonempty, closed, convex subset of $X$. Let $T: X \rightarrow 2^{E^{\prime}}$ be a monotone and lower hemicontinuous set-valued mapping such that, for each $x \in X, T x$ is a nonempty subset of $E^{\prime}$. Suppose further that the set $\left\{x \in X: \inf _{y \in S x} \inf _{v \in T y} \operatorname{Re}\langle v, y-x\rangle<0\right\}$ is relatively open in $X$. Then there exists $x \in X$ such that

$$
x \in S x \quad \text { and } \quad \inf _{u \in T x} \operatorname{Re}\langle u, y-x\rangle \geq 0 \quad \text { for each } y \in S x .
$$

\section{Acknowledgment}

The author is grateful to the reviewer for his/her careful reading and valuable suggestions.

\section{REFERENCES}

[1] H. Ben-El-Mechaiekh, P. Deguire, A. Granas, Une alternative non linéaire en analyse convexe et applications, C. R. Acad. Sci. Paris Sér I Math. 295 (1983), 257-259.

[2] F.E. Browder, The fixed point theory of multi-valued mappings in topological vector spaces, Math. Ann. 177 (1968), 283-301.

[3] D. Chan, J.S. Pang, The generalized quasi-variational inequality problem, Math. Oper. Res. 7 (1982), 211-222.

[4] K. Fan, Fixed-points and minimax theorems in locally convex topological vector spaces, Proc. Nat. Acad. Sci. USA 38 (1952), 121-126.

[5] K. Fan, A generalization of Tychonoff's fixed point theorem, Math. Ann. 142 (1961), 305-310.

[6] K. Fan, A minimax inequality and applications, Inequalities, III (Proc. Third Sympos., Univ. California, Los Angeles, Calif., 1969; dedicated to the memory of Theodore S. Motzkin), Academic Press, New York, 1972, pp. 103-113.

[7] K. Fan, Some properties of convex sets related to fixed point theorems, Math. Ann. 266 (1984), 519-537.

[8] I.L. Glicksberg, A further generalization of the Kakutatni fixed point theorem, with application to Nash equilibrium points, Proc. Amer. Math. Soc. 3 (1952), 170-174.

[9] B. Knaster, C. Kuratowski, S. Mazurkiewicz, Ein Beweis des Fixpunktsatzes für $n$-dimensionale Simplexe, Fund. Math. 14 (1929), 132-137.

[10] M.-H. Shih, K.-K. Tan, Generalized quasi-variational inequalities in locally convex topological vector spaces, J. Math. Anal. Appl. 108 (1985), 333-343.

[11] A. Tychonoff, Ein Fixpunktsatz, Math. Ann. 111 (1935), 767-776.

[12] C.L. Yen, A minimax inequality and its applications to variational inequalities, Pacific J. Math. 97 (1981), $477-481$. 